\title{
Green tea polyphenols attenuate hepatic steatosis, and reduce insulin resistance and inflammation in high-fat diet-induced rats
}

\author{
HONG-MIAO XIA ${ }^{1,2}$, JIN WANG $^{1}$, XIAO-JIE XIE ${ }^{1}$, LI-JUAN XU ${ }^{1}$ and SHI-QI TANG ${ }^{1}$ \\ ${ }^{1}$ Medical Examination Center, ${ }^{2}$ Hubei Key Laboratory of Digestive System Disease, \\ Renmin Hospital of Wuhan University, Wuhan, Hubei 430060, P.R. China
}

Received February 25, 2019; Accepted July 4, 2019

DOI: $10.3892 /$ ijmm.2019.4285

\begin{abstract}
Non-alcoholic fatty liver disease (NAFLD) is characterized by hepatic steatosis, insulin resistance and inflammation; however, the exact pathogenesis of NAFLD is not fully understood. Green tea polyphenols (GTP) exhibit beneficial effects against metabolic syndrome. However, the effect of GTP on NAFLD remains largely unknown. The aim of the present study was to investigate the effects of GTP on NAFLD in high-fat diet (HFD)-induced rats. The NAFLD rat model was induced with a HFD for 8 weeks. A total of 30 adult male Sprague Dawley rats were randomly divided into three groups: i) Normal control group; ii) HFD group; and iii) HFD with GTP group. Hematoxylin and eosin and Oil Red $\mathrm{O}$ analyses were performed. The levels of alanine aminotransferase (ALT), aspartate aminotransferase (AST) and inflammatory cytokines in the serum, as well as oxidative stress markers and hepatic lipids in the liver were measured. In addition, parameters associated with glucose metabolism were also assessed. Western blotting and RT-qPCR were used to determine the expression levels of 5' adenosine monophosphate-activated protein kinase (AMPK). HFD-induced rats exhibited features associated with NAFLD. GTP intervention significantly reduced serum ALT and AST levels. Fasting serum glucose, insulin resistance and hepatic lipid levels were all decreased in the GTP-treated rats. GTP also significantly decreased the levels of TNF- $\alpha$, IL- 6 and malondialdehyde. In contrast, superoxide dismutase levels were increased in the liver. Furthermore, GTP also significantly increased phosphorylation of AMPK and attenuated histopathological changes indicative of injury in liver tissue. GTP has a protective effect on HFD-induced hepatic steatosis, insulin resistance and inflammation, and the underlying mechanism may involve the AMPK pathway.
\end{abstract}

Correspondence to: Professor Shi-Qi Tang, Medical Examination Center, Renmin Hospital of Wuhan University, 99 Zhang Zhidong Road, Wuhan, Hubei 430060, P.R. China

E-mail:wh88063953@qq.com

Key words: hepatic steatosis, insulin resistance, inflammation, adenosine monophosphate kinase, non-alcoholic fatty liver disease

\section{Introduction}

Non-alcoholic fatty liver disease (NAFLD) is a stress-induced liver disease, which is characterized by excess accumulation of lipid droplets in hepatocytes and diffused steatosis without excessive alcohol consumption $(1,2)$. The incidence of NAFLD as well as that of metabolic syndrome is on the increase $(3,4)$. NAFLD is considered a major health burden in adolescents and adults, and is closely associated with obesity, hyperlipidemia and diabetes (5). In addition, it has been reported that NAFLD is associated with cardiovascular diseases (6).

Metabolic disturbance and accumulation of lipids in the liver may result in hepatic steatosis, which further induces insulin resistance and inflammation, subsequently leading to diabetes and cardiovascular complications $(7,8)$. In turn, insulin resistance and inflammation exacerbate hepatic steatosis, thus forming a vicious cycle (5), which eventually develops into non-alcoholic steatohepatitis, cirrhosis and liver cancer (9). Day demonstrated that insulin resistance, oxidative stress and lipid peroxidation are crucial factors for NAFLD (10). Therefore, developing strategies to reduce excessive lipid accumulation in the liver is critical for the treatment of NAFLD and prevention of potential complications (11).

Although several interventions have been proposed for patients with NAFLD, none have demonstrated considerable efficacy for limiting liver damage (12). In recent years, the therapeutic effects of Traditional Chinese Medicine in patients with NAFLD have been demonstrated. Green tea polyphenols (GTP) are the effective components extracted from tea, and primarily include catechins, flavonoids, anthocyanins and phenolic compounds. Previous findings have demonstrated the therapeutic effects of GTP in patients with metabolic syndromes and type 2 diabetes $(13,14)$. It has been reported that GTP regulates the metabolism of glucose and lipids by enhancing glycogen synthesis and inhibiting lipogenesis in hepatocytes (14). In addition, GTP mobilizes and activates endogenous antioxidant mechanisms, improving the activity of enzymes such as superoxide dismutase (SOD), decreasing the levels of lipid peroxide in the plasma, and thus detoxifying the liver during metabolism.

In the present study, the therapeutic effects of GTP in a high-fat diet (HFD)-induced NAFLD rat model were examined, and the underlying mechanisms of GTP were explored. The results of the present study highlight the potential 
therapeutic effects of GTP, and may translate to an effective strategy for protecting the liver in patients with NAFLD.

\section{Materials and methods}

Animals. A total of 30 adult male Sprague Dawley rats (weight, 180-200 g) were obtained from Hunan SJA Laboratory Animal Co., Ltd. The rats were housed at $24^{\circ} \mathrm{C}$ in a standard specific-pathogen-free environment with a 12/12 h light/dark cycle, and were acclimatized for a week. The animal experiments were performed in accordance with The Principles of Laboratory Animal Care (NIH publication no. 85Y23, revised 1996), and all procedures were approved by The Experimental Animal Ethics Committee of Wuhan University Renmin Hospital.

Experimental design and procedures. The rats were randomly assigned to one of following three experimental groups with ten rats in each: i) Normal control group (NC); ii) HFD group; and iii) HFD with GTP group (GTP).

Rats in the HFD and GTP groups were fed HFD, and $\mathrm{NC}$ rats were fed standard laboratory chow for 8 weeks. The HFD was composed of $70 \%$ fat, $20 \%$ carbohydrate, and $10 \%$ protein, with equal quantities of other components, such as fiber, vitamins, and minerals, to the standard chow diet. The GTP rats were administered GTP (Xiya reagent, cat. no. 20875) at $200 \mathrm{mg} / \mathrm{kg}$ daily via oral gavage simultaneously for 8 weeks. The main components of GTP are catechins compounds, which have been identified as catechin, citricin, catechin citrate and catechin epicatechin. The $\mathrm{NC}$ and HFD groups were administered the same volume of saline. All the rats were weighed weekly. The timing and dosage of GTP, which exhibited beneficial effects against liver injury in rats, were determined in our preliminary study (data not shown).

After 8 weeks on the assigned diet/treatment, rats were fasted for $12 \mathrm{~h}$. Rats were anesthetized with isoflurane (induced with 5\% isoflurane and maintaining with $3 \%$ in $21 /$ min oxygen flow in a sealed container). Blood samples were collected from the postcava with vacuum serum separator tubes (BD Vacutainer SST; Becton, Dickinson and Company), and centrifuged at $4^{\circ} \mathrm{C}, 1,500 \mathrm{xg}$ for $10 \mathrm{~min}$. The serum was collected and stored at $-80^{\circ} \mathrm{C}$. Portions of the liver were obtained quickly, washed with ice-cold PBS and subsequently flash frozen in liquid nitrogen and stored at $-80^{\circ} \mathrm{C}$ for the molecular assays. The samples were then preserved in RNA sample preservation solution and stored at $-20^{\circ} \mathrm{C}$ until the RNA was isolated. Alternatively, the samples were fixed in $4 \%$ phosphate-buffered formaldehyde for histological analysis. All the rats were sacrificed by cervical dislocation.

GTT. After fasting for $12 \mathrm{~h}$, the fasting blood glucose and fasting insulin levels of rats were measured using a OneTouch ultra-glucose meter (LifeScan) and ELISA kit (Elabscience), respectively. To perform the GTT, rats were intraperitoneally injected with glucose $(2 \mathrm{~g} / \mathrm{kg}$ body weight), and the blood glucose was measured at $0,15,30,60,90$ and 120 min with a glucometer (15). To reflect the glucose tolerance, the areas under the glucose concentration-time curves of the glucose concentration after glucose injection during the first $120 \mathrm{~min}$ were calculated. Homeostasis model assessment-insulin resistance index (HOMA-IRI) was calculated to evaluate individual insulin resistance levels using the following formula: HOMA-IRI = fasting blood glucose $(\mathrm{mmol} / \mathrm{l}) \times$ fasting insulin $(\mathrm{mIU} / \mathrm{l}) / 22.5$.

Liver function assay and hepatic lipid analyses. Liver function was evaluated by determining the serum concentrations of alanine aminotransferase (ALT) and aspartate aminotransferase (AST) (ALT, Siemens healthineers, cat. no. 03036926 and AST, Siemens healthineers, cat. no. 07499718) using an ADVIA 2400 Chemistry system analyzer. Lipid content of the liver was measured using the corresponding commercial kits for triglyceride (TG), total cholesterol (TC) and non-esterified fatty acid (NEFA) (Nanjing Jiancheng Bioengineering Institute), according to the manufacturers' protocol.

Histopathological examination. Liver tissues were harvested, cut into small pieces, fixed in $4 \%$ paraformaldehyde, embedded with paraffin and subsequently stained with hematoxylin and eosin (H\&E) to visualize histopathological changes of the liver. Alternatively, the tissue samples were embedded with pre-cooled optimal cutting compound for cryostat sectioning and stained with Oil Red O to visualize accumulation of hepatic lipid droplets. Morphology was assessed by two independent pathologists under a light microscope (Olympus BX63; Olympus Corporation). The degree of liver injury was scored according to Kalantar et al (16). Indices including inflammatory cell infiltration, RBC congestion, and nuclear pyknosis were examined. In addition, a semiquantitative analysis was carried out using the following scoring system: 0 , normal; 1 , mild; 2 , moderate; and 3 , severe. The average score for each group was measured to determine the total score. Lipid droplets were quantified as described by Jiang et al (17). Results are presented as area fractions, i.e., the percentage of specific counts in relation to total number of counted points.

Measurement of serum and liver tissue indicators of inflammation. The levels of inflammatory cytokines TNF- $\alpha$ and IL-6 in the serum were quantified with corresponding ELISA kits (Elabscience), according to the manufacturer's protocols. The frozen liver tissues were homogenized and centrifuged at $4^{\circ} \mathrm{C}, 12,000 \mathrm{xg}$ for $20 \mathrm{~min}$, and the supernatants were then collected. The oxidative stress markers malondialdehyde (MDA) and SOD in liver tissues were measured with biochemical assay kits (Nanjing Jiancheng Bioengineering Institute), and all procedures were performed according to the manufacturer's protocol and repeated three times.

$R T-q P C R$. Total RNA was extracted from liver samples using TRIzol ${ }^{\circledR}$ and reverse transcribed to cDNA. RT-qPCR was performed using the Faststart Universal SYBR Green Master (ROX) kit (Roche Diagnostics) in an CFX Connect ${ }^{\mathrm{TM}}$ Real-Time PCR Detection system (Bio-Rad Laboratories). The thermocycling conditions were $95^{\circ} \mathrm{C}$ for $10 \mathrm{mins}$, followed by 40 cycles of denaturation $95^{\circ} \mathrm{C}$ for $15 \mathrm{sec}$ and a combined annealing/extension step at $60^{\circ} \mathrm{C}$ for $1 \mathrm{~min}$. The primer sequences of the target genes were: GAPDH forward, 5'-CGCTAACATCAAATGGGGTG-3' and reverse, 5'-TTGCTGACAATCTTGAGGGAG-3'; and 5' adenosine 
monophosphate-activated protein kinase (AMPK) $\alpha$ forward, 5'-CTCAGGAAGGCTGTATGCGG-3' and reverse, 5'-ACG GTTGAGATACTCCGGGAT-3'. The mRNA expressions levels were normalized to the gene GAPDH and analyzed using the $2^{-\Delta \Delta \mathrm{Cq}}$ method (18). All the reactions were performed in triplicate.

Western blotting. Total protein was extracted from liver samples using a total protein extraction kit (Beyotime Institute of Biotechnology), and the protein concentrations were determined using a bicinchoninic acid assay. A total of $20 \mu \mathrm{g}$ protein was loaded per well, resolved using a $10 \%$ SDS-PAGE gel and transferred to a PVDF membrane. The membranes were blocked with 5\% skimmed milk in TBST buffer (TBS containing $0.1 \%$ Tween-20) at room temperature for $2 \mathrm{~h}$ and subsequently incubated with the following primary antibodies at $4^{\circ} \mathrm{C}$ overnight: Anti-phospho-AMPKa (Abcam; cat. no. ab133448; 1:1,000), anti-AMPK $\alpha$ (Abcam; cat. no. ab80039; 1:1,000) and anti-GAPDH (Abcam; cat. no. ab181602; 1:1,000). After washing with TBST, the blots were incubated with the corresponding horseradish peroxidase-conjugated secondary antibodies (Abcam; goat anti-rabbit antibody, cat. no. ab205718 and goat anti-mouse antibody, ab205719; 1:2,000) at room temperature for $1 \mathrm{~h}$. The immunoreactive bands were imaged with a ChemiDoc XRS system (Bio-Rad Laboratories, Inc.) using enhanced chemiluminescent reagent. The densities of the bands were quantified with Quantity One version 4.6 (Bio-Rad Laboratories, Inc.).

Statistical analysis. All statistical analyses were performed using SPSS software (version 20.0; SPSS, Inc.). Data are presented as the mean \pm standard deviation. Differences among multiple groups were compared with one-way ANOVA followed by Tukey's post hoc test. $\mathrm{P}<0.05$ was considered to indicate a statistically significant difference.

\section{Results}

GTP inhibits weight gain in HFD rats. All the rats were fed the same amount of food per day during the study period and their body weight was monitored weekly. As shown in Fig. 1, the weight of HFD rats gradually increased after 2 weeks and was significantly higher compared with the NC group $(\mathrm{P}<0.05)$. However, when the rats were pretreated with GTP, the weight gain was slower and the weight was significantly lower compared with the HFD rats $(\mathrm{P}<0.05)$ throughout the study period ( 8 weeks).

GTP alleviates insulin resistance induced by HFD. To investigate the effect of GTP on insulin resistance, fasting glucose and insulin levels were measured, and a GTT was performed and the HOMA-IRI was calculated. As presented in Fig. 2A-C, after 8 weeks of HFD, rats developed notably higher fasting glucose and fasting insulin levels, and a higher HOMA-IRI, which was indicative of increased insulin resistance compared with the NC group. GTP intervention inhibited the increase in fasting glucose and fasting insulin levels as well as the HOMA-IRI (both $\mathrm{P}<0.05$ ) in the HFD rats. In addition, as the results of the glucose tolerance test showed, HFD impaired glucose tolerance and the area under

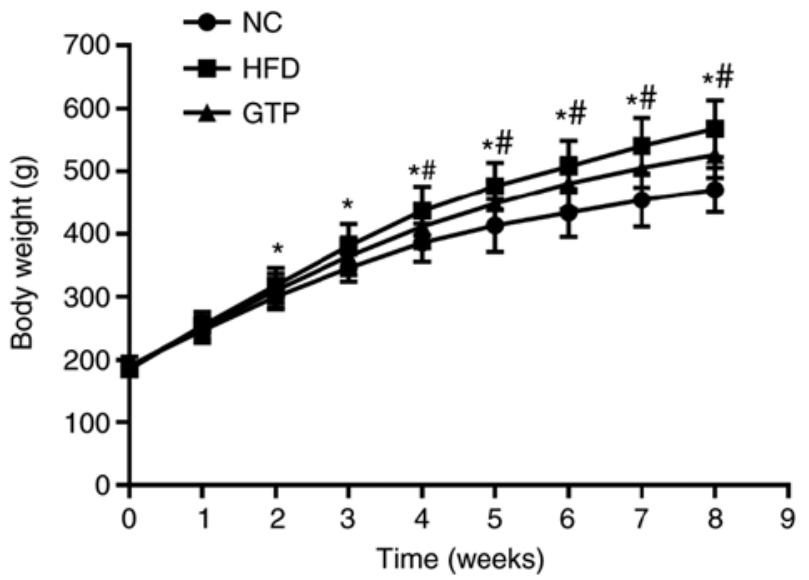

Figure 1. GTP reduces weight gain in the HFD rats. The body weight of rats was monitored weekly ( $\mathrm{n}=10$ rats/group). NC, normal control group; HFD, high fat diet group; GTP, high fat diet with green tea polyphenols treatment group. ${ }^{*} \mathrm{P}<0.05$ vs. $\mathrm{NC} ;{ }^{~} \mathrm{P}<0.05$ vs. HFD.

the curve for glucose in the GTP group was significantly reduced compared with the HFD rats (Fig. 2D and E). Taken together, these results suggest that GTP may improve insulin resistance in the HFD rats.

GTP improves hepatic function. The serum ALT and AST levels were measured to evaluate the effect of GTP on hepatic function. As shown in Fig. 3, within 8 weeks of the HFD, the serum ALT and AST levels in HFD rats were significantly increased compared with the control group. GTP intervention significantly decreased the levels of ALT and AST (both $\mathrm{P}<0.05$ ) compared with the HFD rats (Fig. 3), suggesting that GTP may improve hepatic function.

GTP mitigates HFD-induced hepatic steatosis. A continuous HFD may lead to the excess accumulation of lipid in the liver, which is termed hepatic steatosis and is one of the most prominent features of NAFLD (19). Therefore, the effect of GTP on hepatic steatosis was determined by measuring hepatic lipid metabolism, and by using H\&E and Oil Red O staining. The concentrations of TG, TC and NEFA were significantly increased in livers of the HFD rats compared with the NC rats after 8 weeks of a HFD (Fig. 4A-C). H\&E staining revealed that the substructure of the liver was normal and clear, and the liver lobules were regular in NC rats. By contrast, the livers of the HFD rats presented with widespread lipid vacuoles and the liver cells were damaged (Fig. 5A). Hepatic steatosis and fat droplets were further confirmed by Oil Red O staining, which is a more sensitive and specific method for the detection of hepatic steatosis. The Oil Red O-staining results showed that there were more lipid droplets in the cryosections of the HFD rats compared with the NC rats (Fig. 5C). Treatment with GTP notably reduced the levels of TG, TC and NEFA in the liver (Fig. 4A-C), alleviated hepatic histopathological injuries (Fig. 5B) and reduced the number and volume of lipid droplets (Fig. 5D), suggesting that GTP may prevent lipid accumulation and hepatic steatosis.

GTP attenuates HFD-induced oxidative stress and the inflammatory response. One of the most notable features 

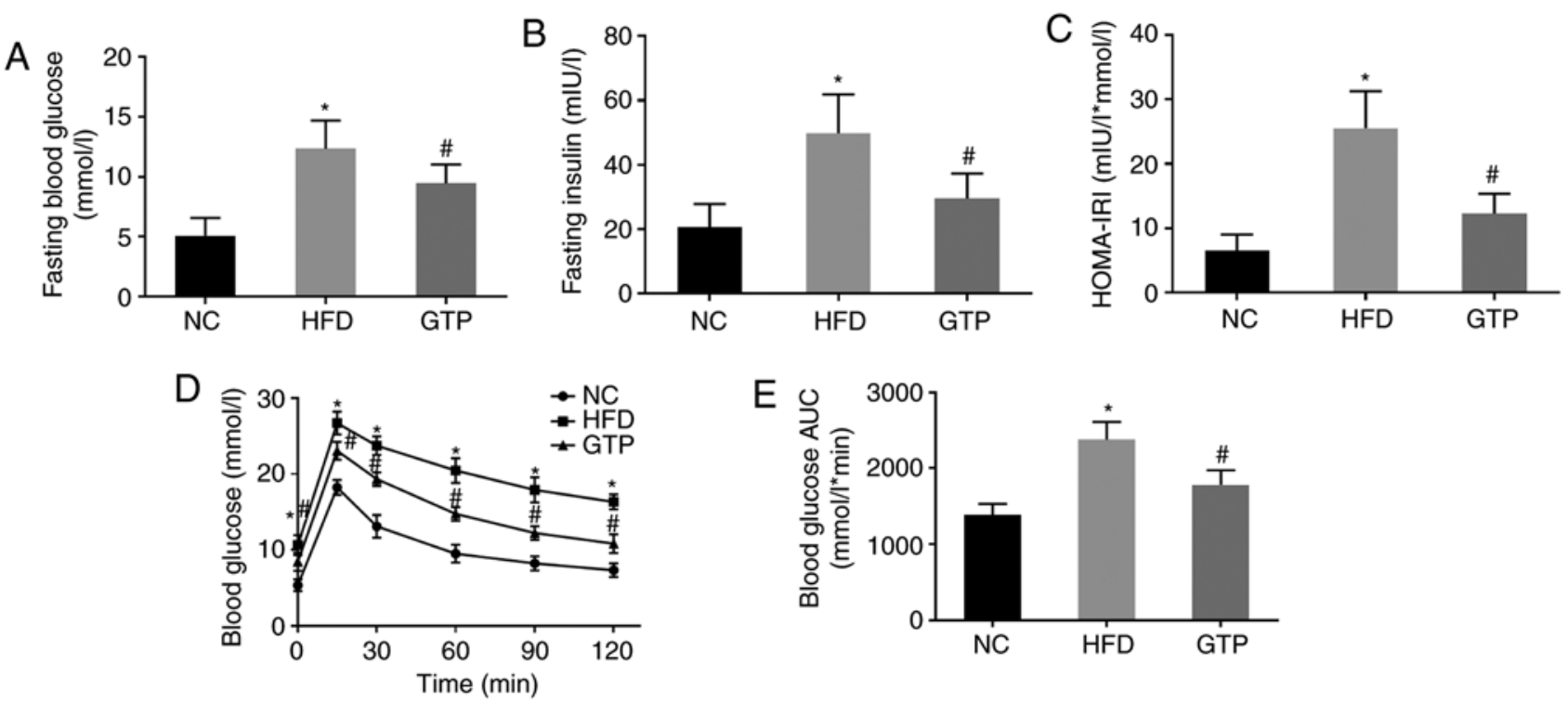

Figure 2. GTP alleviates insulin resistance induced by HFD in rats. (A) Fasting blood glucose levels, (B) fasting insulin levels and (C) HOMA-IRI in rats after 8 weeks of modeling. (D) Glucose tolerance test and (E) the corresponding area under the curve for glucose tolerance test (n=10 rats/group). NC, normal control group; HFD, high fat diet group; GTP, high fat diet with green tea polyphenols treatment group. ${ }^{*} \mathrm{P}<0.05$ vs. NC; ${ }^{*} \mathrm{P}<0.05$ vs. HFD.
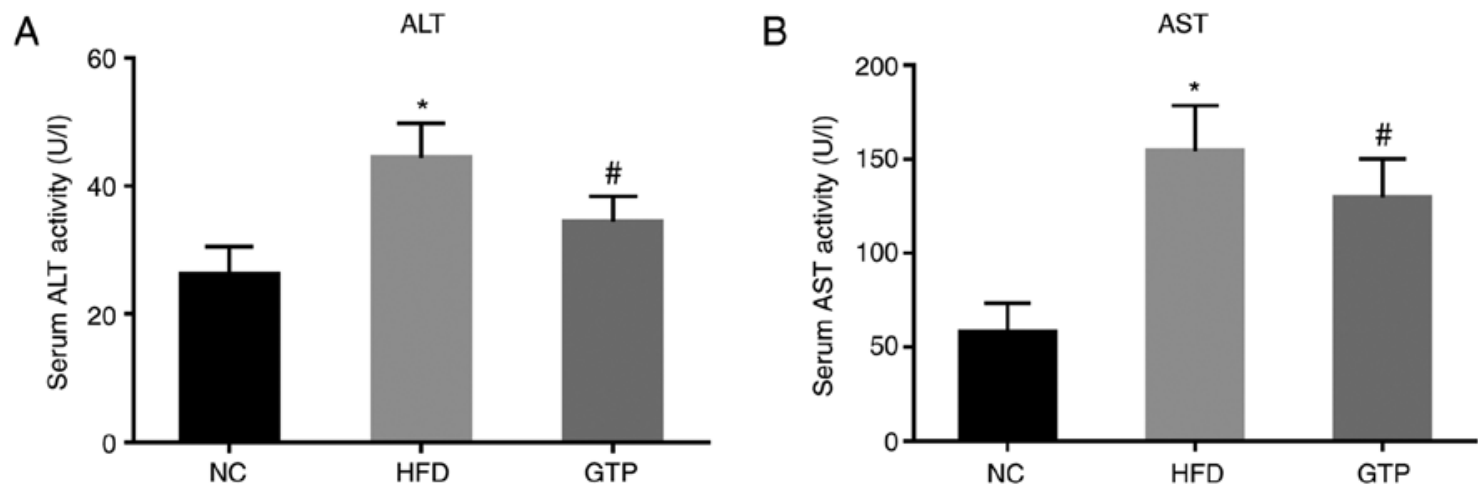

Figure 3. GTP treatment improves hepatic function. (A) Serum alanine transaminase and (B) aspartate transaminase concentrations were measured after 8 weeks of modeling ( $\mathrm{n}=10$ rats/group). NC, normal control group; HFD, high fat diet group; GTP, high fat diet with green tea polyphenols treatment group. ${ }^{*} \mathrm{P}<0.05$ vs. NC; ${ }^{"} \mathrm{P}<0.05$ vs. HFD.
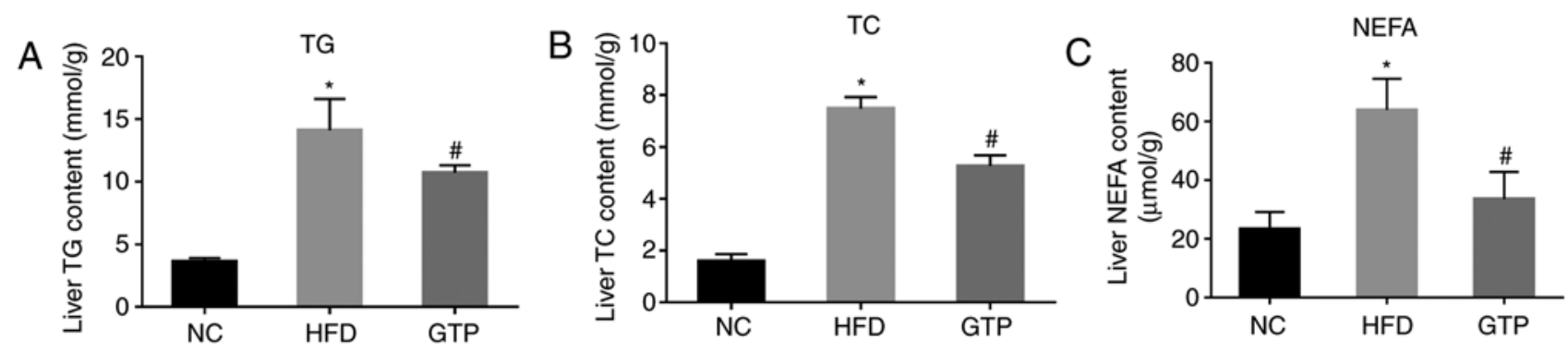

Figure 4. GTP modulates metabolism of hepatic lipids. After 8 weeks of modeling, the levels of (A) TG, (B) TC and (C) NEFA were measured (n=10 rats/group). $\mathrm{NC}$, normal control group; HFD, high fat diet group; GTP, high fat diet with green tea polyphenols treatment group. " $\mathrm{P}<0.05$ vs. NC; ${ }^{~} \mathrm{P}<0.05$ vs. HFD.

of NAFLD is oxidative stress. Therefore, the effect of GTP on oxidative stress was assessed. After 8 weeks of HFD, the level of MDA was significantly increased in the liver of the HFD rats compared with the control group (Fig. 6A), while the activity of SOD was notably decreased (Fig. 6B) and GTP treatment reversed or prevented these changes.
Another important stage of NAFLD is the inflammatory response (20). Therefore, the effect of GTP on HFD-induced inflammatory response was measured using ELISA. The results showed that the serum levels of the inflammatory cytokines TNF- $\alpha$ and IL- 6 were notably increased in the HFD rats compared with the control group, and GTP treatment decreased 
A

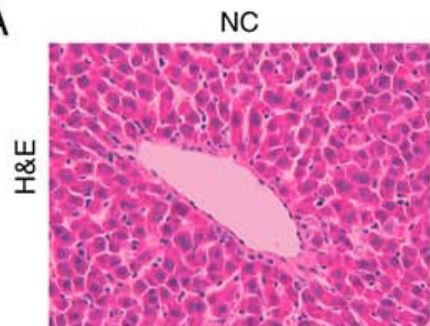

C

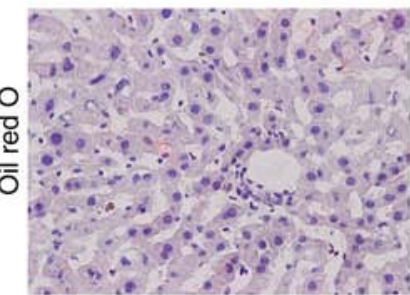

HFD
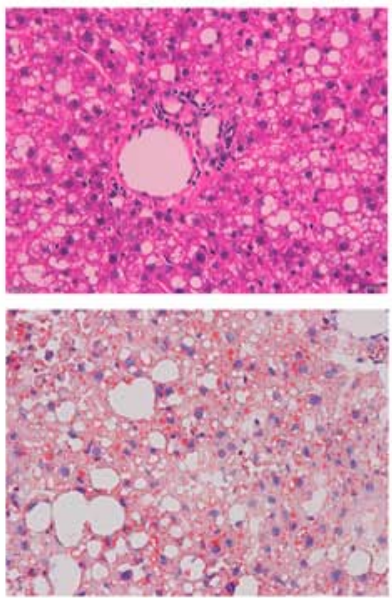

GTP
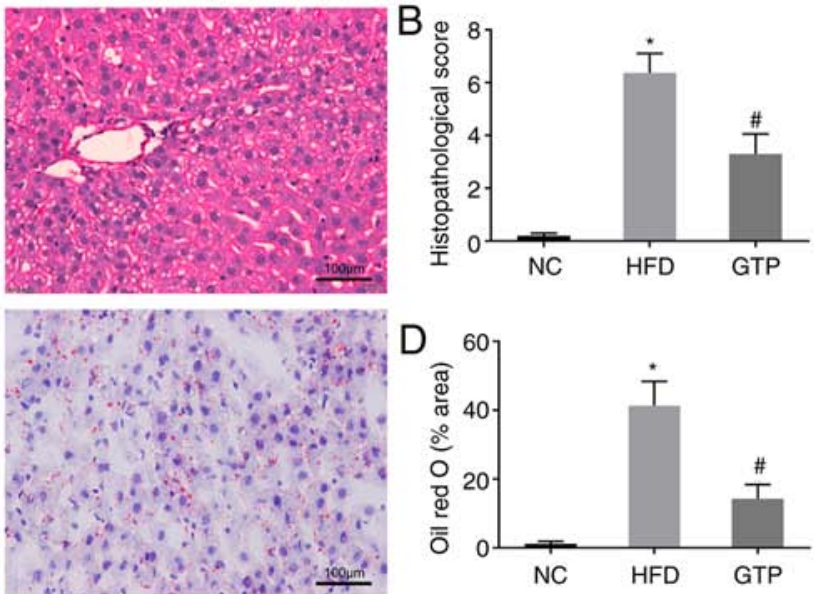

Figure 5. GTP reduces HFD-induced hepatic steatosis. Representative (A) Hematoxylin and eosin (H\&E) and (C) Oil Red O staining of liver samples to visualize hepatic steatosis in liver sections. Scale bar, $100 \mu \mathrm{m}$. Compared with the livers in NC rats, a larger unstained area that consisted of fat vacuoles (H\&E staining) (B) and a larger red area that consisted of lipid droplets (Oil Red O staining) (D) were observed in HFD rats, while GTP treatment notably prevented these changes in the liver ( $\mathrm{n}=10$ rats/group). NC, normal control group; HFD, high fat diet group; GTP, high fat diet with green tea polyphenols treatment group. ${ }^{*} \mathrm{P}<0.05$ vs. $\mathrm{NC} ;{ }^{\text {"P }}<0.05$ vs. HFD.

A

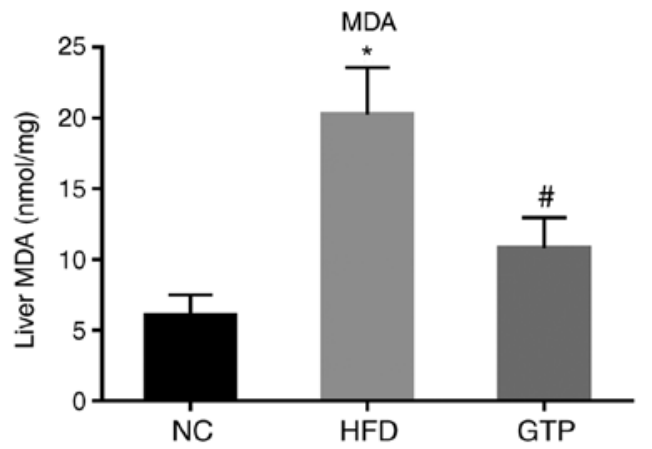

C

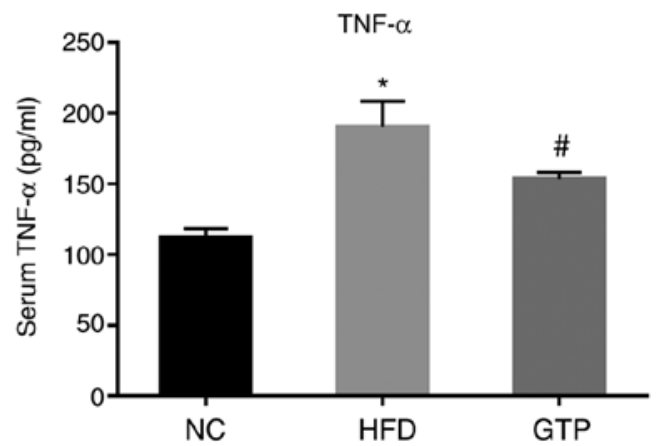

B

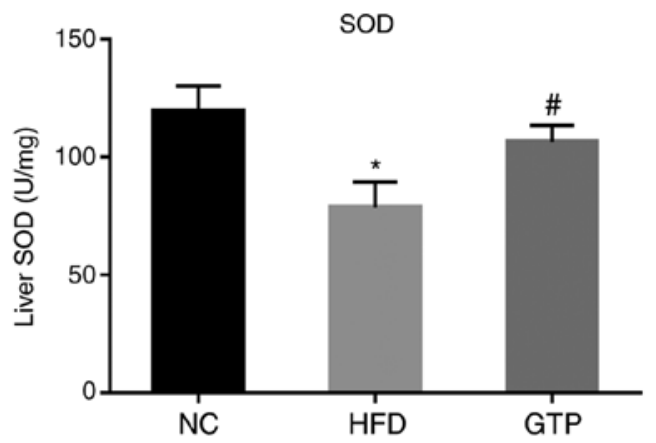

D

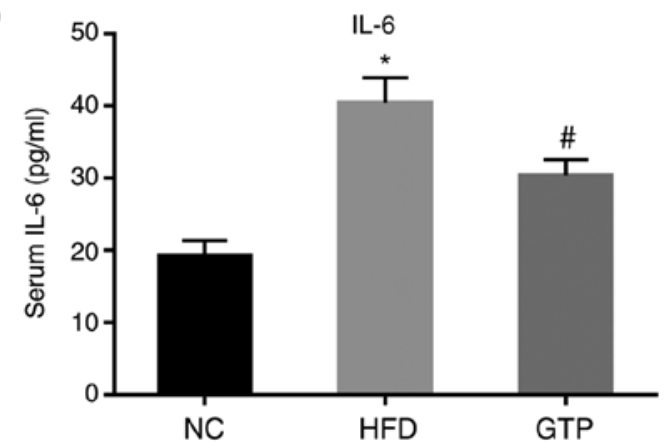

Figure 6. GTP prevents HFD-induced oxidative stress and inflammatory response. The levels of oxidative stress markers (A) MDA and (B) SOD in liver tissues and inflammatory cytokines (C) TNF- $\alpha$, and (D) IL-6 in the serum of each group were quantified ( $\mathrm{n}=10 \mathrm{rats} / \mathrm{group}$ ). Data are represented as mean \pm standard error of the mean and duplicated 3 times. NC, normal control group; HFD, high fat diet group; GTP, high fat diet with green tea polyphenols treatment group. ${ }^{*} \mathrm{P}<0.05$ vs. NC, ${ }^{\#} \mathrm{P}<0.05$ vs. HFD.

the levels of TNF- $\alpha$ and IL-6 in the HFD rats (Fig. 6C and D; both $\mathrm{P}<0.05)$. Taken together, the findings suggest that GTP inhibits HFD-induced oxidative stress and the inflammatory response in the liver of rats.

GTP enhances AMPK activation in the liver of HFD rats. AMPK is one of the key enzymes regulating the metabolism of lipids. Therefore, it was hypothesized that GTP may exert its protective effects by modulating the AMPK signaling pathway. As shown in Fig. 7, western blotting revealed that the levels of AMPK $\alpha$ and phospho-AMPK $\alpha$ were significantly lower in the liver of HFD rats compared with the control rats, and GTP treatment significantly upregulated the levels of AMPK $\alpha$ and phospho-AMPK $\alpha$. Consistent with these results, the mRNA expression levels of AMPK $\alpha$ in liver of HFD rats were significantly lower compared with the NC rats, and GTP 

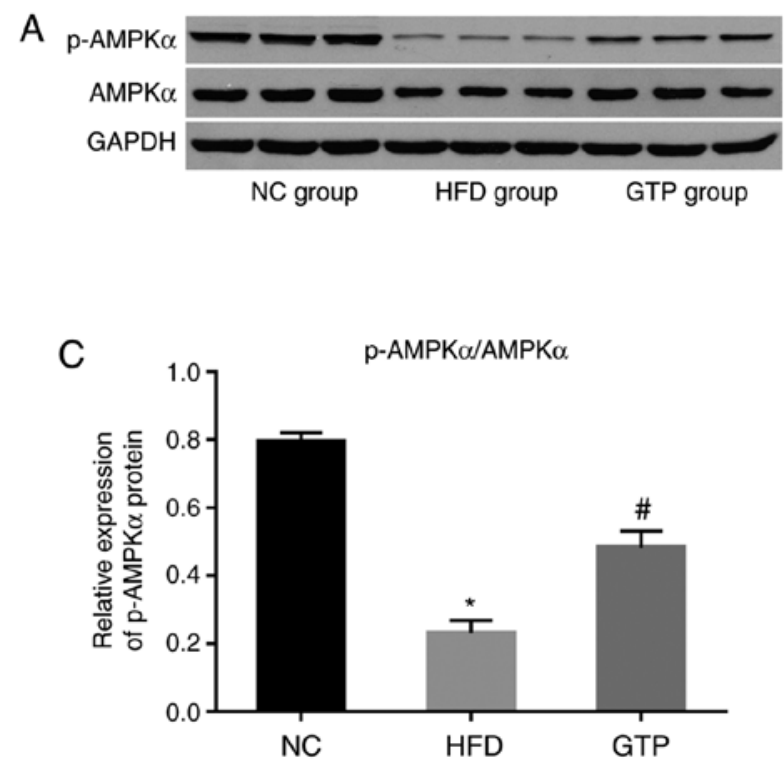
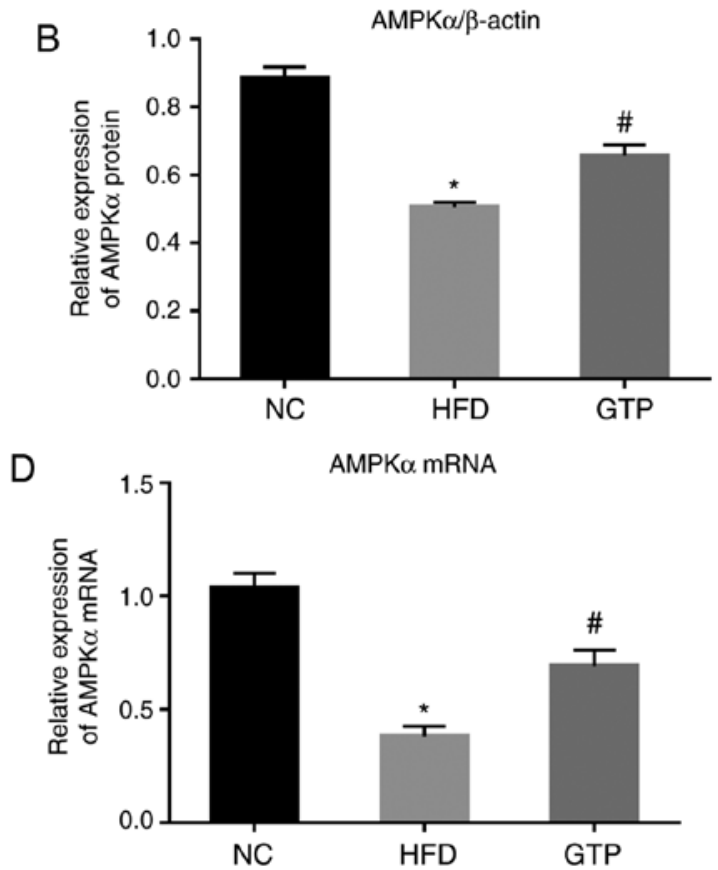

Figure 7. GTP enhances AMPK activity in the liver of HFD rats. (A) Representative western blots of AMPK $\alpha$ and p-AMPKa. (B) Quantification of AMPKa and p-AMPK $\alpha$ expression relative to GAPDH. (C) Ratio of p-AMPK $\alpha$ to AMPK $\alpha$ expression. (D) mRNA expression of AMPK $\alpha$ in the liver. All the results are presented as mean \pm standard error of the mean of three independent experiments ( $\mathrm{n}=10$ rats/group). NC, normal control group; HFD, high fat diet group; GTP, high fat diet with green tea polyphenols treatment group. ${ }^{*} \mathrm{P}<0.05$ vs. NC; ${ }^{*} \mathrm{P}<0.05$ vs. HFD.

treatment increased its expression. These results suggest that the hepatoprotective role of GTP may be mediated by upregulation of the AMPK signaling pathway.

\section{Discussion}

NAFLD is closely associated with obesity, hyperlipidemia and diabetes mellitus. NAFLD is increasingly prevalent and is a leading risk factor of hepatic cirrhosis and liver cancer (21). However, there are no effective therapeutic strategies for treating NAFLD at present owing to an incomplete understanding of the underlying molecular mechanisms. In the present study, the effectiveness of GTP treatment in protecting against HFD-induced NAFLD in rats was demonstrated. Treatment with GTP alleviated obesity, hepatic steatosis, insulin resistance and inflammation in HFD-induced NAFLD rats. The results suggest that GTP may serve as a promising therapeutic treatment for prevention of NAFLD and metabolic syndrome.

In the present study, HFD-fed rats were used to mimic the key metabolic characteristics of humans with NAFLD. After 8 weeks of a HFD, the NAFLD rat model was successfully established as evidenced by the significantly increased weight of HFD rats, elevated ALT and AST levels, hepatic steatosis, insulin resistance and inflammation, which are known to aggravate the progression of NAFLD $(22,23)$. Therefore, the effects of GTP on these conditions were examined to determine its effects on NAFLD.

GTP intervention significantly inhibited the weight gain of HFD rats, and rats in the GTP group weighed significantly less compared with the rats in the HFD group, suggesting that GTP may reduce obesity. Furthermore, GTP treatment significantly improved hepatic function, indicated by ALT and AST levels, and decreased the levels of TG, TC and NEFA in the liver of HFD rats. The elevated serum ALT and AST levels are the primary abnormalities reported in patients with NAFLD and other hepatic diseases (24). Histopathological analysis showed that GTP also reduced the number and volume of lipid droplets and reduced the HFD-induced histopathological changes in the liver, which indicates that GTP protects against HFD-induced hepatic steatosis.

The excess accumulation of lipids in the body and organs promotes oxidative stress and induces an inflammatory response, which are considered the two primary causes of pathogenesis of NAFLD (25). Oxidative stress and lipid peroxidation are part of the second stage of NAFLD. Oxidative stress is the consequence of the imbalance between the generation of reactive oxygen species and the antioxidant defense responses (26). SOD and MDA are the primary indicators of oxidative stress. The activity of SOD indirectly reflects the capacity to remove oxygen-free radicals, whereas MDA, a lipid peroxidation product, reflects the degree of lipid peroxidation and indirectly represents the degree of hepatic cell injury. In the present study, after 8 weeks of HFD, the activity of SOD in the liver was significantly decreased, whereas the content of MDA was increased. In addition, GTP treatment significantly enhanced the activity of SOD and decreased the levels of MDA, indicating that GTP can inhibit oxidation through the upregulation of SOD activity and downregulation of MDA content. However, the exact mechanism of how GTP upregulates SOD activity and downregulates MDA content need to be further explored.

Previous findings have confirmed that the inflammatory response is another stage of NAFLD. IL-6 and TNF- $\alpha$ are the most common cytokines in the inflammatory response. IL-6 is regarded as a potential mediator leading to NAFLD, and TNF- $\alpha$ 
has been implicated in liver fibrosis and the advanced stages of NAFLD in humans $(27,28)$. In the present study, HFD-induced NAFLD rats exhibited significantly higher serum TNF- $\alpha$ and IL-6 levels, and the changes were reversed by GTP treatment. In agreement with the present study, a previous study reported that epigallocatchin-3-gallate, a major active compound of GTP, may protect against HFD-induced liver inflammation in rats (29). The results suggest that GTP treatment may effectively prevent or attenuate the HFD-induced inflammatory response in the liver of rats.

In addition to anti-oxidative stress and inflammatory response effects, GTP also reduced insulin resistance in HFD-induced NAFLD rats. The elevated levels of inflammatory cytokines inhibit insulin signaling and result in insulin resistance $(30,31)$. Along with the inflammatory response, HFD-induced rats presented significantly upregulated fasting glucose and insulin levels, as well as HOMA-IRI, indicating that the rats developed insulin resistance. GTP treatment alleviated these pathophysiological changes as evidenced by the decreased fasting glucose and insulin levels.

In NAFLD, owing to increased adipose lipolysis, excess free fatty acids/NEFAs are delivered to the liver and converted into TGs, leading to the accumulation of lipids and thus lipotoxicity to hepatocytes (32). In the present study, NEFA levels were significantly increased in the liver of HFD rats when compared with the control, and after 8 weeks of GTP treatment, the elevated NEFA levels were significantly decreased, which may reduce the delivery of NEFAs to the liver for hepatic TG synthesis and accumulation, thus preventing lipid peroxidation-induced hepatic injury $(32,33)$. Furthermore, reduction of hepatic lipids may be attributed to improved hyperglycemia, as GTP treatment decreased glucose influx to the liver and thus ultimately decreased lipogenesis in the liver.

AMPK serves a critical role in the regulation of hepatic lipogenesis $(34,35)$ and it is also involved in the insulin signaling pathway. AMPK is activated through promoting its phosphorylation at Thr172 (36), and further induces phosphorylation of acteyly-CoA-carboxylase (ACC), causing a reduction in ACC activity, which leads to a reduction in hepatic TG synthesis $(37,38)$. In the present study, the level of phosphorylated AMPK in the liver of rats exposed to HFD was significantly reduced compared with the NC rats, and GTP treatment significantly increased phosphorylation of AMPK. However, how GTP upregulates AMPK activity needs to be further explored. The lack of the identification of the underlying mechanism is a limitation to the present study. Further experiments should be conducted to clarify the mechanism.

In conclusion, the results of the present study suggest that GTP treatment may protect against HFD-induced NAFLD in rats by inhibiting hepatic steatosis, insulin resistance and inflammation through enhancing AMPK activity. These findings represent promising therapeutic possibilities of GTP for the treatment of NAFLD and related metabolic pathologies in human.

\section{Acknowledgements}

The authors are grateful to the Hubei Key Laboratory of Digestive System Disease of Renmin Hospital of Wuhan
University for providing relevant experimental facilities and technical support.

\section{Funding}

No funding was received.

\section{Availability of data and materials}

The datasets used and/or analyzed during the current study are available from the corresponding author on reasonable request.

\section{Authors' contributions}

HMX and SQT contributed to the conception and design of the study. HMX performed the experiments and wrote the manuscript. JW and XJX established the rat model and administered the GTP treatment. HMX, JW and XJX performed ELISA, western blotting and reverse transcription-quantitative PCR analyses. LJX analyzed the data. All authors read and approved the final manuscript.

\section{Ethics approval and consent to participate}

Animal use and care were in accordance with The Principles of Laboratory Animal Care (NIH publication no. 85Y23, revised 1996) and all procedures were approved by the Experimental Animal Ethics Committee of Wuhan University Renmin Hospital.

\section{Patient consent for publication}

Not applicable.

\section{Competing interests}

The authors declare that they have no competing interests.

\section{References}

1. Lee JH, Baek SY, Jang EJ, Ku SK, Kim KM, Ki SH, Kim CE, Park KI, Kim SC and Kim YW: Oxyresveratrol ameliorates nonalcoholic fatty liver disease by regulating hepatic lipogenesis and fatty acid oxidation through liver kinase B1 and AMP-activated protein kinase. Chem Biol Interact 289: 68-74, 2018.

2. Chalasani N, Younossi Z, Lavine JE, Charlton M, Cusi K, Rinella M, Harrison SA, Brunt EM and Sanyal AJ: The diagnosis and management of nonalcoholic fatty liver disease: Practice guidance from the American Association for the Study of Liver Diseases. Hepatology 67: 328-357, 2018.

3. Williams CD, Stengel J, Asike MI, Torres DM, Shaw J, Contreras M, Landt CL and Harrison SA: Prevalence of nonalcoholic fatty liver disease and nonalcoholic steatohepatitis among a largely middle-aged population utilizing ultrasound and liver biopsy: A prospective study. Gastroenterology 140: 124-131, 2011.

4. Wong VW, Chu WC, Wong GL, Chan RS, Chim AM, Ong A, Yeung DK, Yiu KK, Chu SH, Woo J, et al: Prevalence of non-alcoholic fatty liver disease and advanced fibrosis in Hong Kong Chinese: A population study using proton-magnetic resonance spectroscopy and transient elastography. Gut 61: 409-415, 2012.

5. Luo P, Qin C, Zhu L, Fang C, Zhang Y, Zhang H, Pei F, Tian S, Zhu XY, Gong J, et al: Ubiquitin-specific peptidase 10 (USP10) inhibits hepatic steatosis, insulin resistance, and inflammation through Sirt6. Hepatology 68: 1786-1803, 2018. 
6. Anstee QM, Targher G and Day CP: Progression of NAFLD to diabetes mellitus, cardiovascular disease or cirrhosis. Nat Rev Gastroenterol Hepatol 10: 330-344, 2013.

7. Samuel VT, Liu ZX, Qu X, Elder BD, Bilz S, Befroy D, Romanelli AJ and Shulman GI: Mechanism of hepatic insulin resistance in non-alcoholic fatty liver disease. J Biol Chem 279: 32345-32353, 2004

8. Savage DB, Petersen KF and Shulman GI: Disordered lipid metabolism and the pathogenesis of insulin resistance. Physiol Rev 87: 507-520, 2007.

9. El-Sherbiny M, Eldosoky M, El-Shafey M, Othman G, Elkattawy HA, Bedir T and Elsherbiny NM: Vitamin D nanoemulsion enhances hepatoprotective effect of conventional vitamin D in rats fed with a high-fat diet. Chem Biol Interact 288 65-75, 2018.

10. Day CP: Non-alcoholic fatty liver disease: A massive problem. Clin Med (Lond) 11: 176-178, 2011.

11. Ding S, Jiang J, Zhang G, Bu Y,Zhang G and Zhao X: Resveratrol and caloric restriction prevent hepatic steatosis by regulating SIRT1-autophagy pathway and alleviating endoplasmic reticulum stress in high-fat diet-fed rats. PLoS One 12: e0183541, 2017.

12. Rotman Y and Sanyal AJ: Current and upcoming pharmacotherapy for non-alcoholic fatty liver disease. Gut 66: 180-190, 2017.

13. Sabu MC, Smitha K and Kuttan R: Anti-diabetic activity of green tea polyphenols and their role in reducing oxidative stress in experimental diabetes. J Ethnopharmacol 83: 109-116, 2002.

14. Kim JJ, Tan Y, Xiao L, Sun YL and Qu X: Green tea polyphenol epigallocatechin-3-gallate enhance glycogen synthesis and inhibit lipogenesis in hepatocytes. Biomed Res Int 2013: 920128 2013.

15. Lubaczeuski C, Gonçalves LM, Vettorazzi JF, Kurauti MA, Santos-Silva JC, Bonfleur ML, Boschero AC, Costa-Júnior JM and Carneiro EM: Vagotomy reduces insulin clearance in obese mice programmed by low-protein diet in the adolescence. Neura Plast 2017: 9652978, 2017.

16. Kalantar M, Kalantari H, Goudarzi M, Khorsandi L, Bakhit S and Kalantar H: Crocin ameliorates methotrexate-induced liver injury via inhibition of oxidative stress and inflammation in rats. Pharmacol Rep 71: 746-752, 2019.

17. Jiang S, Tang X, Wang K, Liang Y, Qian Y, Lu C and Cai L: Hepatic functional and pathological changes of type 1 diabetic mice in growing and maturation time. J Cell Mol Med: Jun 20, 2019 (Epub ahead of print). doi.org/10.1111/jcmm.14504.

18. Livak KJ and Schmittgen TD: Analysis of relative gene expression data using real-time quantitative PCR and the 2(-Delta Delta C(T)) method. Methods 25: 402-408, 2001.

19. Tilg $H$ and Moschen AR: Insulin resistance, inflammation, and non-alcoholic fatty liver disease. Trends Endocrinol Metab 19: 371-379, 2008

20. Gong J, Fang C, Zhang P, Wang PX, Qiu Y, Shen LJ, Zhang L, Zhu XY, Tian S, Li F, et al: Tumor progression locus 2 in hepatocytes potentiates both liver and systemic metabolic disorders in mice. Hepatology 69: 524-544, 2019.

21. Zhao GN, Zhang P, Gong J, Zhang XJ, Wang PX, Yin M, Jiang Z, Shen LJ, Ji YX, Tong J, et al: Tmbim1 is a multivesicular body regulator that protects against non-alcoholic fatty liver disease in mice and monkeys by targeting the lysosomal degradation of Tlr4. Nat Med 23: 742-752, 2017.
22. Kitade H, Chen G, Ni Y and Ota T: Nonalcoholic fatty liver disease and insulin resistance: New insights and potential new treatments. Nutrients 9: pii: E387, 2017.

23. Samuel VT and Shulman GI: Mechanisms for insulin resistance: Common threads and missing links. Cell 148: 852-871, 2012.

24. Arora A and Sharma P: Non-invasive diagnosis of fibrosis in non-alcoholic fatty liver disease. J Clin Exp Hepatol 2: 145-155, 2012.

25. Podrini C, Borghesan M, Greco A, Pazienza V, Mazzoccoli G and Vinciguerra M: Redox homeostasis and epigenetics in non-alcoholic fatty liver disease (NAFLD). Curr Pharm Des 19: 2737-2746, 2013.

26. Reddy VP, Zhu X, Perry G and Smith MA: Oxidative stress in diabetes and Alzheimer's disease. J Alzheimers Dis 16: 763-774, 2009.

27. Kishimoto T: IL-6: From its discovery to clinical applications. Int Immunol 22: 347-352, 2010.

28. Lesmana CR, Hasan I, Budihusodo U, Gani RA, Krisnuhoni E Akbar N and Lesmana LA: Diagnostic value of a group of biochemical markers of liver fibrosis in patients with non-alcoholic steatohepatitis. J Dig Dis 10: 201-206, 2009.

29. Xiao J, Ho CT, Liong EC, Nanji AA, Leung TM, Lau TY, Fung ML and Tipoe GL: Epigallocatechin gallate attenuates fibrosis, oxidative stress, and inflammation in non-alcoholic fatty liver disease rat model through TGF/SMAD, PI3 K/Akt/FoxO1, and NF-kappa B pathways. Eur J Nutr 53: 187-199, 2014

30. Wen H, Ting JP and O'Neill LA: A role for the NLRP3 inflammasome in metabolic diseases-did Warburg miss inflammation? Nat Immunol 13: 352-357, 2012.

31. Park MH, Kim DH, Lee EK, Kim ND, Im DS, Lee J, Yu BP and Chung HY: Age-related inflammation and insulin resistance: A review of their intricate interdependency. Arch Pharm Res 37: 1507-1514, 2014

32. Guturu P and Duchini A: Etiopathogenesis of nonalcoholic steatohepatitis: Role of obesity, insulin resistance and mechanisms of hepatotoxicity. Int J Hepatol 2012: 212865, 2012.

33. Jones JG: Hepatic glucose and lipid metabolism. Diabetologia 59 1098-1103, 2016

34. Lambert JE, Ramos-Roman MA, Browning JD and Parks EJ: Increased de novo lipogenesis is a distinct characteristic of individuals with nonalcoholic fatty liver disease. Gastroenterology 146: 726-735, 2014.

35. Smith BK, Marcinko K, Desjardins EM, Lally JS, Ford RJ and Steinberg GR: Treatment of nonalcoholic fatty liver disease: Role of AMPK. Am J Physiol Endocrinol Metab 311: E730-E740, 2016.

36. Chiu CY, Chan IL, Yang TH, Liu SH and Chiang MT: Supplementation of chitosan alleviates high-fat diet-enhanced lipogenesis in rats via adenosine monophosphate (AMP)-activated protein kinase activation and inhibition of lipogenesis-associated genes. J Agric Food Chem 63: 2979-2988, 2015.

37. Cho YS, Lee JI, Shin D, Kim HT, Jung HY, Lee TG, Kang LW, Ahn YJ, Cho HS and Heo YS: Molecular mechanism for the regulation of human ACC2 through phosphorylation by AMPK. Biochem Biophys Res Commun 391: 187-192, 2010.

38. Choi S, Choi Y, Choi Y, Kim S, Jang J and Park T: Piperine reverses high fat diet-induced hepatic steatosis and insulin resistance in mice. Food Chem 141: 3627-3635, 2013. 